\title{
Intelligent Reflecting Surface-assisted mmWave Communication with Lens Antenna Array
}

\author{
Yazheng Wang, Hancheng Lu, Dan Zhao, Yansha Deng and Arumugam Nallanathan
}

\begin{abstract}
In millimeter wave (mmWave) communication, lens antenna array has been considered as a potential technique to combat the severe path loss. However, blockage effect still exists. To address this issue, we design an intelligent reflecting surface (IRS) assisted wideband mmWave communication system with lens antenna array with limited radio frequency (RF) chains. This is the first attempt to design a mmWave communication system with the integration of IRS and lens antenna array. The designed system can realize high robust, low-complexity and costeffective mmWave communication. To achieve desired performance, we first derive the signal-to-interference-plus-noise ratio (SINR) expression with the intersymbol interference, then we formulate a SINR maximization problem, by jointly considering the beamforming vector at base station with limited RF chains and reflection coefficient matrices at IRS with discrete phase levels. This problem is intractable and non-convex. To solve it, we optimize the reflection coefficient matrices by quadratic transformation with low-complexity closed-form expression, and the beamforming vector is handled by sparse beamforming optimization, where the $l_{0}$ norm is approximated by the $l_{1}$ norm. Finally, simulation results demonstrate the advantages of our proposed algorithm compared with benchmark schemes, e.g., 5 dB SINR gain can be achieved compared with existing algorithm.
\end{abstract}

Index Terms - Intelligent reflecting surface (IRS), lens antenna array, millimeter wave (mmWave), blockage effect, beamforming.

\section{INTRODUCTION}

Millimeter Wave (mmWave) communication, with frequency ranging from $30 \sim 300 \mathrm{GHz}$ to provide Gbps data rate, is regarded as a key technology for fifth generation $(5 \mathrm{G})$ mobile communication and beyond [1]-[3]. Due to the higher carrier frequency, mmWave suffers from orders-of-magnitude more free-space-loss path loss compared with the lower frequency bands [4]. Fortunately, smaller wavelengths of mmWave can be more effective by compacting large number of antennas at either the transmitter or the receiver to achieve significant beamforming gains via compensating for the severe path loss.

Despite the benefits brought by the large antenna array to mmWave, it also suffers from high complexity in computation and high cost in energy consumption, since each antenna requires one dedicated radio frequency $(\mathrm{RF})$ chain in conventional multiple input multiple output (MIMO) communication

Y. Wang, H. Lu, D. Zhao are with the Information Network Laboratory, Department of Electronic Engineering and Information Science, University of Science and Technology of China, Hefei 230027, China (e-mail: hclu@ustc.edu.cn; \{wang1997, zd2019\}@mail.ustc.edu.cn).

Y. Deng is with King's College London, London, UK (email: yansha.deng@kcl.ac.uk).

A. Nallanathan is with the School of Electronic Engineering and Computer Science, Queen Mary University of London, London, UK (e-mail: a.nallanathan@qmul.ac.uk). system [6], [7]. A potential way to realize low complex and cost-effective mmWave communication is to use lens antenna array [8]-[16], which was first proposed in [11] based on the concept of beamspace MIMO communication [12]. Specifically, it is generally consists of an electromagnetic (EM) lens and a matching antenna array located in the focal region of the EM lens [8], [10], and is capable of changing the propagation directions of the EM rays, analogous to the optical lenses. By manipulating the direction of the EM rays, lens antenna array embraces angle-dependent energy focusing property. With this unique property and the angular sparsity of mmWave channels, mmWave signals can be approximately transformed from antenna space to beamspace. Since the beam dimension of mmWave signals is much smaller than antenna dimension, the required number of RF chains in lens antenna array can be significantly reduced [13] to enable the low-complexity and cost-effective mmWave communication. Existing works mainly focus on the performance of lens antenna array [8][17]. Zeng et al. in [8], [10] proposed a cost-effective mmWave lens system for the single-user and multi-user scenario. In [14], Wang et al. proposed a spectrum-efficient and energyefficient mmWave transmission scheme, where NOMA was integrated to support more users in beamspace mmWave communication systems. Due to the limited RF chains constraint at BS, generally a small set of antennas were chosen for data transmission. In [15], Guo et al. considered the joint design of beam selection and precoding matrices to maximize the sum-rate of a downlink single-sided lens MU-MIMO mmWave system, while an efficient algorithm was proposed based on the innovative penalty dual decomposition method. Huang et al. [16] studied the joint beamforming and antenna selection problem for the downlink multi-user mmWave lens antenna array system.

However, with lens antenna array, mmWave communication still suffers from blockage effect, which severely degrades the system performance. Large antenna array usually forms narrow transmission beams, which is suitable for fixed lineof-sight (LoS) links. Such links are vulnerable to blockage effect, which frequently happens due to the obstacles in the environments. Particularly, due to the narrow beamwidth of mmWave, even a small misalignment between the transmitter and receiver antennas or a very small obstacle, such as a person's arm, will significantly block the link [4], [33]. Meanwhile, due to the time-varing mmWave channels and the strong path loss of mmWave signals, the performance enhancement brought by lens antenna array is still limited and the optimization of BS beamforming is insufficient for a satisfying communication quality. 
To overcome blockage effect and establish robust mmWave connection when the LoS link and non-LoS links with natural refections are blocked by obstacles [29], [31], [33], we introduce intelligent reflecting surface (IRS) into the mmWave lens system. IRS is a recently proposed promising technology, as a kind of reconfigurable intelligent surfaces that can reconfigure the wireless propagation environment via softwaredefined reflection [18]-[33]. In detail, IRS is a planar array consisting of a large number of reconfigurable low-cost passive elements, where each element can independently reflect the incident signals with reconfigurable amplitudes and phase shifts through the aid of the smart micro controller [18]. By properly adjusting the passive beamforming, the signals reflected by IRS with different paths can be constructively added at the receiver to enhance the desired signals power, cancel the undesired signals, and suppress interferences [21]. In [20], Guo et al. focused on the maximization of the weighted sum-rate, while the beamforming at BS was optimized based on the fractional programming method. Wu et al. [21] proposed to minimize the total BS transmit power by jointly optimizing active beamforming and passive beamforming under the quality of service constraint of each user. In [22], Huang et al. focused on the energy efficiency issue and provided up to $300 \%$ energy efficiency compared with using conventional multi-antenna amplify-and-forward relay. On the other hand, channel estimation in IRS-assisted systems is a challenging problem since IRS is composed of passive reflecting elements and is unable to perform signal processing [23]-[27]. A least square channel estimation scheme was proposed in [23], where the elements were serially switched on. You et al. [24] designed a novel hierarchical training reflection with discrete IRS phase levels. In [26], the channel estimation in IRS-assisted mmWave was cast as a sparse signal recovery problem and solved by compressed-sensing methods. Moreover, IRS is applicable to mmWave systems due to its reconfigurability and low hardware cost [28]-[33]. Wang et al. in [28] proposed to maximize the received signal power in IRS-assisted mmWave systems by exploiting some important characteristics of mmWave channels. Zuo et al. investigated the combination of IRS, NOMA and mmWave to further enhance the sum-rate performance of wireless system [29]. In [30], Cao et al. studied the power minimization problem in IRS-assisted uplink mmWave system where time delay requirements were taken into consideration. However, these existing works considered mmWave system with massive transmit antennas and RF chains, which leads to unaffordable energy consumption and hardware cost.

Motivated by this, we attempt to utilize the significant advantages of both lens antenna array and IRS to enhance the system performance without incurring huge energy consumption. With the potential to achieve high robust, low-complexity and cost-effective wideband mmWave communication, integrating IRS with lens antenna array brings the following unique challenges. First, the detrimental multi-path effect in wideband communications, i.e., the intersymbol interference (ISI) should be taken into account. Furthermore, the expressions of the ISI and the signal-to-interference-plus-noise ratio (SINR) are different from existing works only with lens an- tenna array or IRS, making it challenging to perform optimization to obtain the desired performance. Second, as stated in [8]-[10], [16], the massive antennas in mmWave system lead to unaffordable computation complexity. For practical designs that need to operate with low signal processing complexity and limited RF chains, the traditional ISI mitigation method such as orthogonal frequency division multiplexing (OFDM) becomes less appealing and effective [9], [10]. Therefore, we exploit IRS to enhance the received signal power and weaken the ISI simultaneously without incurring additional signal processing for OFDM. Third, different from the conventional uniform linear array (ULA) and uniform planar array (UPA), lens antenna array with limited RF chains is equipped at base station (BS), which leads to a non-convex $l_{0}$ norm constraint and brings the need to jointly design the beamforming and the antenna selection. Futhermore, we consider mulitple IRS with discrete phase levels, which is more practical than continuous phase levels. As a result, the optimization becomes more complex than existing works [20], [21], [28].

In this paper, we attempt to combat aforementioned challenges by leveraging the benefits from IRS and lens antenna array. We design a multi-IRS assisted wideband mmWave communication system with lens antenna array, which can achieve high robust, low-complexity, and cost-effective mmWave communication. Specifically, lens antenna array is exploited at BS to reduce the RF chains cost and multiple IRS are deployed to circumvent the obstacles and enhance the system performance by properly optimizing the IRS reflection coefficient matrices. Therefore, the main contributions of this paper can be summarized as follows:

- To reduce the hardware complexity and energy consumption, we exploit lens antenna array in mmWave system. Meanwhile, IRS is utilized to overcome blockage effect and enhance the system performance. To the best of our knowledge, we are the first to design such IRS and lens antenna array integrated wideband mmWave communication system.

- For practical designs with low processing complexity and limited RF chains, the conventioal ISI mitigation methods such as OFDM becomes less effective. Therefore, we utilize IRS to improve the received signal power as well as weaken the ISI. We first derive the SINR expression with the ISI, then we formulate the SINR maximization problem, by jointly considering the beamforming vector at BS with limited RF chains and the reflection coefficient matrices at IRS with discrete phase levels.

- To solve such an intractable and non-convex problem, we propose an efficient two-step optimization algorithm. The IRS reflection coefficient matrices optimization is solved by quadratic transformation. Specifically, all the elements in IRS reflection coefficient matrices are optimized with low-complexity closed-form expression serially. The BS beamforming vector optimization is handled by sparse beamforming optimization under the limited RF chain constraint, where the non-convex $l_{0}$ norm is approximated by $l_{1}$ norm.

- Finally, extensive simulations are performed to evaluate 
the performance of our proposed algorithm. The simulation results demonstrate that our proposed algorithm converges fast and achieves significant performance gains compared with benchmark schemes, e.g., $5 \mathrm{~dB}$ SINR gain can be achieved compared with existing algorithm.

The rest of this paper is organized as follows. In Section II we present the related technologies of the designed system. In Section III, the system model is described, then the maxmin problem with joint active and passive beamforming optimization is formulated. In Section IV the formulated problem is carefully analyzed and solved. Performance evaluation is conducted in Section V. Finally, we conclude this paper in Section VI.

Notations: Scalars are denoted by italic letters, vectors and matrices are presented by bold-face lower-case and uppercase letters, respectively. For any general matrix $\boldsymbol{A}, a_{i, j}$ is the $i$-th row and $j$-th column element. $\boldsymbol{A}^{T}, \boldsymbol{A}^{*}$ and $\boldsymbol{A}^{H}$ represent the transpose, conjugate, and conjugate transpose of $\boldsymbol{A}$, respectively. For any general complex-valued vector $\boldsymbol{x}$, $\boldsymbol{x}^{*}$ represents its conjugate, $x_{i}$ denotes the $i$-th element of $\boldsymbol{x}$, and $\operatorname{diag}\{\boldsymbol{x}\}$ denotes a diagonal matrix with each diagonal element being the corresponding element in $\boldsymbol{x} .\|\boldsymbol{x}\|_{0},\|\boldsymbol{x}\|_{1}$, $\|\boldsymbol{x}\|,\|\boldsymbol{x}\|_{\infty}$ denote $\ell_{0}, \ell_{1}, \ell_{2}$ and $\ell_{\infty}$ norm of the vector $\boldsymbol{x}$, respectively. $\mathbb{C}^{x \times y}$ represents the space of $x \times y$ complexvalued matrices. $\operatorname{sinc}(\cdot)$ is the "sinc" function defined as $\operatorname{sinc}(x) \triangleq \sin (\pi x) /(\pi x) \cdot \operatorname{Re}\{\cdot\}$ denotes the real part of a complex number. The calligraphy upper-case letter $\mathcal{M}$ denotes a set.

\section{System Model and Problem Formulation}

We consider a multi-IRS assisted downlink single-user mmWave lens MISO system with one user served by one BS, as illustrated in Fig. 1. The BS is equipped with lens antenna array with $M$ antenna elements, and the user is equipped with only one antenna element. Taking into account the costeffective implementations, we assume that the BS only has $M_{R F} \mathrm{RF}$ chains. These $M_{R F} \mathrm{RF}$ chains are connected to the $M$ antenna elements with analog switches. Since the mmWave links are highly susceptible to environmental blockages and dynamics, we assume the direct mmWave link between the BS and the user is severely blocked by obstacles, such that the BS can only communicate with the user through the signals reflected by the IRSs.

\section{A. Multi-IRS assisted downlink mmWave lens transmission}

The multi-IRS assisted downlink mmWave lens link is a concatenation of three components, i.e., the BS-IRS link, IRS reflection coefficient matrix , and the IRS-user link. We deploy $L$ IRS between BS and user, and each IRS corresponds to one main path with path delay $\tau_{l}$. Without loss of generality, those subpaths that are indistinguishable in neither temporal nor spatial domain are grouped into a single main path [16].

In our designed system, each IRS is assumed to comprise $N$ elements. Each element of the IRS receives the signal from a specific path, then these elements independently reflect the incident signal by controlling its amplitude and phase to achieve passive beamforming. All these distributed IRSs are managed by a smart controller, which can exchange channel state information (CSI) and coordinate the reflecting modes for all IRSs. Meanwhile, due to the high path loss of mmWave and the distributed deployment of multiple faraway IRSs, the inter-IRS links for cooperative IRSs are ignored, which is similar to [28], [31]. At the user side, signals reflected by different IRSs may be superimposed to enhance the received power or cause inter symbol interference, corresponding to their respective path transmission delay. We assume the CSI is perfectly known at the BS and IRS ${ }^{1}$, which is the same as [20], [21].

\section{B. Multi-IRS assisted mmWave lens channel model}

Due to the additional space gap required between the EM lens and antenna elements, lens antenna array is more inclined to be deployed at the BS [10]. Under general multi-path communication environments, the channel impulse response from the $m$-th antenna element of the BS to the user can be expressed as

$$
\theta_{m}(t)=\sum_{l=1}^{L} \boldsymbol{g}_{l}^{H}(t) \boldsymbol{\Phi}_{l} \boldsymbol{h}_{m, l}(t),
$$

where $L$ represents the number of spatial paths that can be distinguished by the $m$-th antenna. We assume that each path is assisted by one IRS. $\boldsymbol{h}_{m, l}(t) \in \mathbb{C}^{N \times 1}$ denotes the channel impulse response between the $m$-th antenna and the $l$-th IRS. $\Phi_{l} \in \mathbb{C}^{N \times N}$ denotes the diagonal reflection coefficient matrix of the $l$-th IRS. $\boldsymbol{g}_{l}^{H}(t) \in \mathbb{C}^{1 \times N}$ denotes the channel impulse response between the $l$-th IRS and the user.

The reflection coefficient matrix can be expressed as $\boldsymbol{\Phi}_{l}=$ $\operatorname{diag}\left\{\varrho_{l, 1} e^{j \varphi_{l, 1}}, \varrho_{l, 2} e^{j \varphi_{l, 2}} \cdots, \varrho_{l, N} e^{j \varphi_{l, N}}\right\}$, where $j$ represents the imaginary unit. For simplicity, we assume the amplitude reflection coefficient $\varrho_{l, N}=1, \forall l, n$ throughout this paper. In practice, the reflection element only has finite reflection levels. Similar to [20], [22], in this paper we consider that $\varphi_{l, n}$ only takes $2^{b}$ discrete values, where $b$ represents the phase resolution in number of bits.

The channel impulse response $\boldsymbol{h}_{m, l}$ can be obtained as

$$
\boldsymbol{h}_{m, l}(t)=\beta_{l} \alpha_{m}^{*}\left(\phi_{l}\right) \boldsymbol{a}_{l}\left(\phi_{l}^{\prime}\right) \delta\left(t-\bar{\tau}_{l}\right),
$$

where $\beta_{l}$ denotes the complex-valued path gain; $\phi_{l}$ is the angle of departure (AoD) of the $l$-th path, and $\bar{\tau}_{l}$ represents the path delay of the $l$-th path between BS and IRS. $\boldsymbol{a}_{l}\left(\phi_{l}^{\prime}\right) \in \mathbb{C}^{N \times 1}$ in (2) denotes the array response of the IRS with the angle of arrival (AoA) $\phi_{l}^{\prime}$, and $\alpha_{m}^{*}\left(\phi_{l}\right)$ denotes the response function of $m$-th antenna with the AoD $\phi_{l}$, which can be expressed as

$$
\alpha_{m}\left(\phi_{l}\right)=e^{-j \phi_{0}} \sqrt{A} \operatorname{sinc}\left(m-\tilde{\Gamma} \sin \left(\phi_{l}\right)\right), m \in \mathcal{M},
$$

where $\phi_{0}$ is a common phase shift from the lens' aperture to the array. $\tilde{\Gamma} \triangleq \Gamma_{y} / \lambda$ represents the lens dimension, which along the azimuth plane that normalized by the carrier wavelength $\lambda . \mathcal{M} \triangleq\left\{0, \pm 1, \cdots, \pm \frac{M-1}{2}\right\}$ denotes the set of

\footnotetext{
${ }^{1}$ Though this is an idealistic assumption, it is still meaningful to study the performance gains brought by IRS for the mmWave lens system. How to obtain CSI at IRS is out of the scope of this paper, and some related works can be found in [23]-[27].
} 


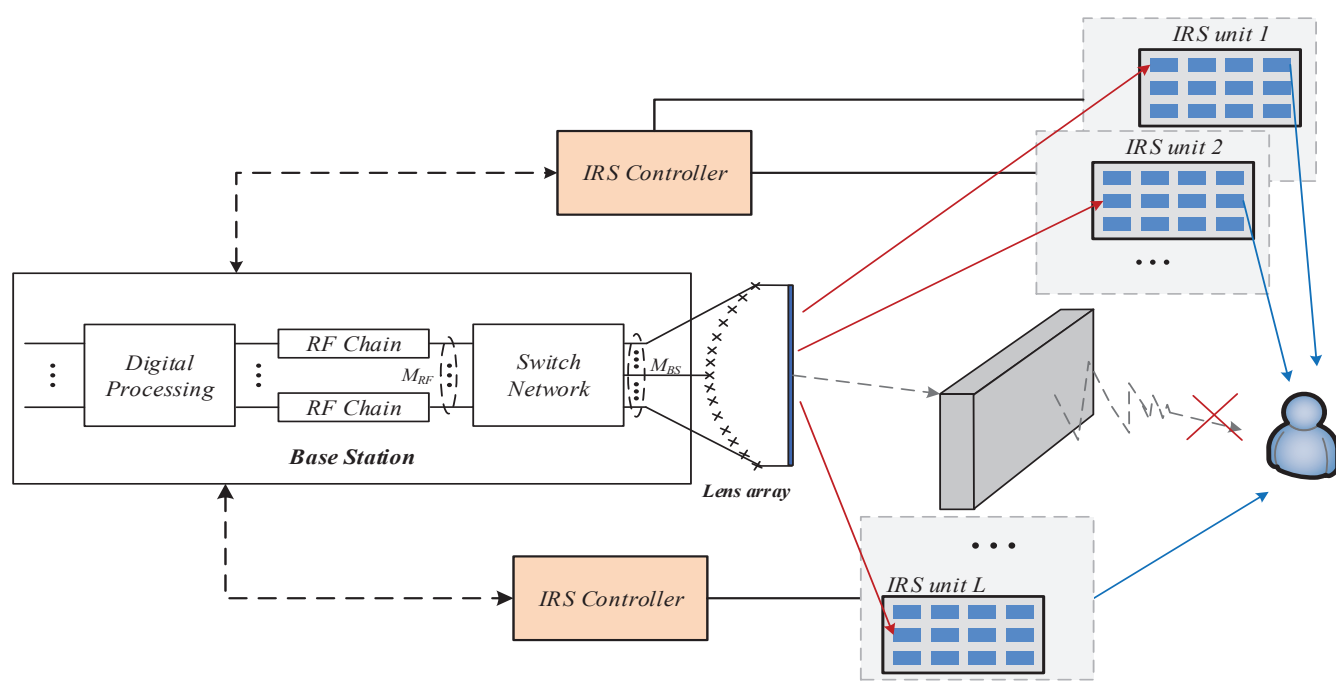

Fig. 1. Proposed intelligent reflecting surface assisted millimeter wave communication with lens antenna array.

antenna indices. The number of antenna elements $M$ depends on $\tilde{\Gamma}$, i.e., $M=1+2[\tilde{\Gamma}\rfloor$, which means more antenna elements should be deployed for larger lens dimension on $\tilde{\Gamma}[8]$. The antenna elements are placed on the focal arc of lens with $\sin \left(\psi_{m}\right)=\frac{m}{\tilde{\Gamma}}$, where $\psi_{m} \in[-\pi / 2, \pi / 2]$ represents the angle of $m$-th antenna element relative to the horizontal axis.

Due to the angle-dependent energy focusing property of lens antenna array and the angular sparsity of mmWave channel, only a small set of antenna elements located in the close vicinity of the energy focusing point steers significant power to the user.

The channel impulse response $g_{l}$ can be modeled as

$$
\boldsymbol{g}_{l}=\sum_{g=1}^{G_{p}} \rho_{l, g} \boldsymbol{a}_{l}\left(\varpi_{l, g}\right) \delta\left(t-\vartheta_{l}\right)
$$

where $G_{p}$ is the number of paths and $\rho_{l, g}$ represents the complex-valued path gain. The array response of the $l$-th IRS with AoD $\varpi_{l, g}$ is denoted by $\boldsymbol{a}_{l}\left(\varpi_{l, g}\right)$, and the total path delay of the $l$-th path associated with the $l$-th IRS can be otained by $\tau_{l}=\bar{\tau}_{l}+\vartheta_{l}$.

\section{Problem formulation}

Without loss of generality, let $T_{s}$ denote the symbol duration. Meanwhile, we assume the multi-path delays $\left\{\tau_{l}\right\}$ are integer multiple of $T_{s}$ [10], [16], i.e., $\tau_{l}=n_{l} T_{s}$ with integers $n_{l}, \forall l$. The path delay can be efficiently obtained by the methods in [10], [17]. Hence we follow the assumption that perfect time synchronization can be achieved at BS, i.e., $\tau_{l}$ can be acquired at BS [8], [16]. Then we can express the time-discrete equivalent channel impulse response function as

$$
\theta_{m}[n]=\sum_{l=1}^{L} \theta_{m, l} \delta\left[n-n_{l}\right], m \in \mathcal{M},
$$

where $\theta_{m, l} \triangleq \boldsymbol{g}_{l}^{H} \boldsymbol{\Phi}_{l} \beta_{l} \alpha_{m}^{H}\left(\phi_{l}\right) \boldsymbol{a}_{l}\left(\phi_{l}^{\prime}\right)$. Actually, $\left|\theta_{m, l}\right|^{2}$ represents the power gain from the $m$-th antenna to the user through the $l$-th spatial path. For each antenna $m$ and user , let $l_{m} \in\{1, \cdots, L\}$ represents the strongest spatial path among all $L$ paths which will be discussed later.
Intuitively, when the signals propagating through the strongest paths arrive at the user simultaneously, ISI will be inherently mitigated. Thus, at each antenna $m$, path delay pre-compensation technique is applied based on its strongest spatial path $l_{m}$ [10], [16]. In this case, the transmitted signals can be expressed as

$$
x_{m}[n]=p_{m} s\left[n+n_{l_{m}}\right], m \in \mathcal{M},
$$

where $p_{m}$ denotes the beamforming coefficient at the $m$-th antenna for the user. $n_{l_{m}}$ is the delay pre-compensation of the strongest path.

Actually, the path delay pre-compensation is the shift of the input symbol with respect to the transmission path delay of the strongest path from the $m$-th antenna element to the user. This operation can be readily implemented at the BS before the baseband precoding. Therefore, at the receiver side, the received signal of the user can be represented as

$$
\begin{aligned}
y[n] & =\sum_{m \in \mathcal{M}} \sum_{l=1}^{L}\left[\sum_{n=1}^{N} g_{l, n} e^{j \varphi_{l, n}} h_{m, l, n}\right] x_{m}\left[n-n_{l}\right]+z[n] \\
& =\sum_{m \in \mathcal{M}} \sum_{l=1}^{L} \boldsymbol{g}_{l}^{H} \boldsymbol{\Phi}_{l} \boldsymbol{h}_{m, l} x_{m}\left[n-n_{l}\right]+z[n],
\end{aligned}
$$

where $z[n]$ represents the additive white Gaussian noise with noise power $\sigma^{2}$. The $n$-th element of $\boldsymbol{g}_{l}^{H}$ and $\boldsymbol{h}_{m, l}$ are denoted by $g_{l, n}$ and $h_{m, l, n}$.

Through separating the strongest path $l_{m}$ on each antenna $m$, (7) can be expressed as

$$
\begin{aligned}
y[n] & =\sum_{m \in \mathcal{M}} \boldsymbol{g}_{l_{m}}^{H} \boldsymbol{\Phi}_{l_{m}} \boldsymbol{h}_{m, l_{m}} x_{m}\left[n-n_{l_{m}}\right] \\
& +\sum_{m \in \mathcal{M}} \sum_{l=1, l \neq l_{m}}^{L} \boldsymbol{g}_{l}^{H} \boldsymbol{\Phi}_{l} \boldsymbol{h}_{m, l} x_{m}\left[n-n_{l}\right]+z[n] .
\end{aligned}
$$

By substituting (6) into (8), the received signals of user can 
be expressed as

$$
\begin{aligned}
y[n] & =\underbrace{\sum_{m \in \mathcal{M}} \boldsymbol{g}_{l_{m}}^{H} \boldsymbol{\Phi}_{l_{m}} \boldsymbol{h}_{m, l_{m}} p_{m} s[n]}_{S_{0}} \\
& +\underbrace{\sum_{m \in \mathcal{M}} \sum_{l=1, l \neq l_{m}}^{L} \boldsymbol{g}_{l}^{H} \boldsymbol{\Phi}_{l} \boldsymbol{h}_{m, l} p_{m} s\left[n-\Delta_{l, l_{m}}\right]}_{S_{1}}+z[n],
\end{aligned}
$$

where $S_{0}$ represents the desired signal, $S_{1}$ represents the inter symbol interference. Let $\Delta_{l, l_{m}} \triangleq n_{l}-n_{l_{m}}$ denote the excessive path delay between the $l$-th path of the user and the strongest path $l_{m}$ of the user. Let $v$ represent the maximum path delay of all paths, i.e., $0 \leq n_{l} \leq v, \forall l$. Therefore, we can obtain $\Delta_{l, l_{m}} \in\{0, \pm 1, \ldots, \pm v\}$. To derive the SINR expression, paths with the same delay should be combined. For any $i \in\{0, \pm 1, \cdots, \pm v\}$, we define

$\theta_{m}[i]= \begin{cases}\boldsymbol{g}_{l}^{H} \mathbf{\Phi}_{l} \boldsymbol{h}_{m, l}, & \text { if } \exists l \in\{1, \cdots, L\}, \text { s.t. } n_{l}-n_{l_{m}}=i ; \\ 0, & \text { otherwise. }\end{cases}$

Based on (10), (9) can be equivalently written as

$$
\begin{aligned}
y[n]= & \underbrace{\sum_{m \in \mathcal{M}} \theta_{m}[0] p_{m} s[n]}_{S_{0}} \\
& +\underbrace{\sum_{i=-v, i \neq 0}^{v} \sum_{m \in \mathcal{M}} \theta_{m}[i] p_{m} s[n-i]}_{S_{1}}+z[n] .
\end{aligned}
$$

By concatenating the signals of all antennas in $\mathcal{M}$, (11) can be further compactly written as

$$
y[n]=\underbrace{\boldsymbol{\Theta}^{H}[0] \boldsymbol{p} s[n]}_{S_{0}}+\underbrace{\sum_{i=-v, i \neq 0}^{v} \boldsymbol{\Theta}^{H}[i] \boldsymbol{p} s[n-i]}_{S_{1}}+z[n],
$$

where $\Theta^{H}[i] \in \mathbb{C}^{1 \times M}$ represents the effective MISO channel impulse response for the signals transmitted that arrive at user with path delay $i$, and $\boldsymbol{p} \in \mathbb{C}^{M \times 1}$ denotes the BS beamforming vector for user. We now derive the SINR of user as

$$
\operatorname{SINR}=\frac{\left|\Theta^{H}[0] p\right|^{2}}{\sum_{i=-v, i \neq 0}^{v}\left|\Theta^{H}[i] p\right|^{2}+\sigma^{2}} .
$$

Note that the numerator of (13) represents the signals reflected by the IRS that arrived at user, through the strongest paths of all the $M$ antennas. Meanwhile, the ISI in the denominator is caused by the signals propogating through the remaining paths.

There are $M_{R F} \leq M \mathrm{RF}$ chains equipped at the BS, and only $M_{R F}$ antennas can be used for transmission at each time. Therefore, antenna selection should be applied at the BS first before transmission. In the mmWave lens single-user system, all beamforming coefficients on this antenna should be set as zero, i.e., $p_{m}=0$, for the purpose of deactivating a specific antenna $m$. In this case, $\boldsymbol{p}$ should have a sparsity structure when less than $M_{R F}$ antennas are activated. Consequently, for the purpose to activate no more than $M_{R F}$ antennas, we have $\|\boldsymbol{p}\|_{0} \leq M_{R F}$.

To ensure the performance in the single-user scenario, we aim to maximize the SINR of the user. Based on our analytical work, the maximization problem can be formulated as

$$
\begin{aligned}
\max _{\left\{\boldsymbol{\Phi}_{l}\right\}_{l=1}^{L}, \boldsymbol{p}} & \frac{\left|\boldsymbol{\Theta}^{H}[0] \boldsymbol{p}\right|^{2}}{\sum_{i=-v, i \neq 0}^{v}\left|\boldsymbol{\Theta}^{H}[i] \boldsymbol{p}\right|^{2}+\sigma^{2}} \\
\text { s.t. } & \|\boldsymbol{p}\|^{2} \leq P_{s}, \\
& \|\boldsymbol{p}\|_{0} \leq M_{R F} . \\
& \varphi_{l, n} \in\left\{0, \frac{2 \pi}{2^{b}}, \frac{2 \pi * 2}{2^{b}}, \ldots, \frac{2 \pi *\left(2^{b}-1\right)}{2^{b}}\right\},
\end{aligned}
$$

where $P_{s}$ is the transmit power constraint at the BS. Problem (14) is an intractable non-convex problem since both (14c) and (14d) are non-convex. Specifically, (14c) corresponds to the BS beamforming vector with limited RF chains. Meanwhile, the discrete phase levels constraint in (14d) makes the problem more complicated.

\section{Problem Analysis And Solution}

In this section, we aim to solve the problem (14) by performing the analysis via two-step optimization.

\section{A. Problem Analysis}

1) Path selection: For each antenna element at the BS, there will be multiple spatial paths that can be chosen for data transmission. As presented in Section III, each antenna will choose the strongest path for data transmission. However, how to choose this strongest path has not been clarified, especially when the IRS reflection coefficient matrices are involved. To make the problem tractable, we first present the path selection criterion described as Lemma 1, which facilitates the joint optimization of BS beamforming vector and IRS reflection coefficient matrices. This low-complexity path selection strategy is suboptimal, yet sufficient for achieving excellent system performance.

Lemma 1: The strongest path between the $m$-th antenna and the user should maximize $\left(\sum_{n=1}^{N}\left|g_{l, n} h_{m, l, n}\right|\right)^{2}$, i.e., $l_{m}=$ $\arg \max _{l=1, \ldots, L}\left(\sum_{n=1}^{N}\left|g_{l, n} h_{m, l, n}\right|\right)^{2}$.

Proof: Let us first revisit the SINR expression (13) of user. It can be seen that, by adjusting the phase of IRS that is associated with the $m$-th antenna, the value of the $m$-th element in $\Theta^{H}[0]$ can be changed. Suppose that the $l$-th path is selected for the $m$-th antenna, and we have $\left|\theta_{m}^{H}[0]\right|^{2}=\left|\boldsymbol{g}_{l}^{H} \boldsymbol{\Phi}_{l} \boldsymbol{h}_{m, l}\right|^{2}=\left|\sum_{n=1}^{N} g_{l, n} h_{m, l, n} e^{j \varphi_{l, n}}\right|^{2}$. Intuitively, $g_{l, n} h_{m, l, n} e^{j \varphi_{l, n}}$ can be seen as that the angel of $g_{l, n} h_{m, l, n}$ rotated by $\varphi_{l, n}$. Let $e^{j \varphi_{l, n}^{*}}=\left(\frac{g_{l, n} h_{m, l, n}}{\left|g_{l, n} h_{m, l, n}\right|}\right)^{*}$, then we can get $\left|\sum_{n=1}^{N} g_{l, n} h_{m, l, n} e^{j \varphi_{l, n}}\right|^{2}=\left(\sum_{n=1}^{N}\left|g_{l, n} h_{m, l, n}\right|\right)^{2}$. 
Obviously, this is the maximum value that $\theta_{m}[0]$ can achieve by changing the angel of $g_{l, n} h_{m, l, n}$ with continuous $\varphi_{l, n}^{*}$, and the range of $\left|\theta_{m}[0]\right|^{2}$ is $0 \leq\left|\theta_{m}[0]\right|^{2} \leq\left(\sum_{n=1}^{N}\left|g_{l, n} h_{m, l, n}\right|\right)^{2}$. In other words, for different path selection strategies, the difference of their influences on SINR is the upper limit of $\left|\theta_{m}[0]\right|^{2}$. Obviously, the larger of the upper limit, the larger of the SINR can be achieved in the following optimization. Therefore, the best path selection strategy is to choose the path that can maximize $\left(\sum_{n=1}^{N}\left|g_{l, n} h_{m, l, n}\right|\right)^{2}$.

According to Lemma 1 , we can select the proper path for each antenna. In the following analysis, we assume that the optimal path has been determined. Hence, the path index $l$ is omitted.

2) Antenna Indices Transformation: In order to construct the explicit expression of SINR about IRS reflection coefficient matrices $\left\{\boldsymbol{\Phi}_{l}\right\}_{l=1}^{L}$, we further define the following antenna indices sets:

$$
\begin{aligned}
\mathcal{M}_{l}[i]=\left\{m \in \mathcal{M} \mid n_{l}-n_{l_{m}}=i\right\}, & \forall i \in\{0, \pm 1, \cdots, \pm v\}, \\
\forall l & \in\{1,2, \cdots, L\} .
\end{aligned}
$$

According to the above defination, it is obvious that we can summarize the following two remarks:

Remark 1: For one given path $l$ and all the $M$ antennas at $\mathrm{BS}$, the excessive path delay betwenn path $l$ and path $l_{m}$ ranges from $-v$ to $v$. That is to say, we can obtain the set of antenna indices $\mathcal{M}$ by merging the sets from $i=-v$ to $i=v$ $: \bigcup_{i=-v}^{v} \mathcal{M}_{l}[i]=\mathcal{M}, \forall l \in\{1,2, \cdots, L\}$. This remark is used to $i=-v$
divide the antenna indices set $\mathcal{M}$ into several subsets, which helps to understand the definition of $\mathcal{M}_{l}[i]$.

Remark 2: For one given excessive path delay $i$, we define the antenna indices set of all the none zero elements in $\mathrm{g}^{H}[i]$ as $\mathcal{M}_{n z}[i]$. Then we can obtain this set by merging the sets from $l=1$ to $l=L: \bigcup_{l=1}^{L} \mathcal{M}_{l}[i]=\mathcal{M}_{n z}[i]$. With the aid of this remark, we can transform the SINR expression (13) to an explicit expression of SINR about $\left\{\boldsymbol{\Phi}_{l}\right\}_{l=1}^{L}$ for further optimization,

\section{B. IRS reflection coefficient matrices optimization}

In this subsection, we consider to optimize the reflection coefficient matrices $\left\{\boldsymbol{\Phi}_{l}\right\}_{l=1}^{L}$, with given BS beamforming vector $\boldsymbol{p}$, where $\boldsymbol{\Phi}_{l}$ denotes the IRS beamforming matrix on the $l$-th path from the $m$-th antenna to the user, where $\boldsymbol{\Phi}_{l}=\operatorname{diag}\left\{e^{j \varphi_{l, 1}}, e^{j \varphi_{l, 2}}, \cdots, e^{j \varphi_{l, N}}\right\}$. For the phase elements of any matrix $\boldsymbol{\Phi}_{l}$, we define $\mathcal{F}=$ $\left\{\varphi_{l, n} \mid \varphi_{l, n} \in\left\{0, \frac{2 \pi}{2^{b}}, \frac{2 \pi * 2}{2^{b}}, \cdots, \frac{2 \pi *\left(2^{b}-1\right)}{2^{b}}\right\}\right\}$.

When BS beamforming vectors $\boldsymbol{p}$ is fixed, problem (14) can be expressed as

$$
\begin{aligned}
& \max _{\left\{\boldsymbol{\Phi}_{l}\right\}_{l=1}^{L}} \frac{\left|\Theta^{H}[0] \boldsymbol{p}\right|^{2}}{\sum_{i=-v, i \neq 0}^{v}\left|\Theta^{H}[i] \boldsymbol{p}\right|^{2}+\sigma^{2}} \\
& \text { s.t. } \varphi_{l, n} \in\left\{0, \frac{2 \pi}{2^{b}}, \frac{2 \pi * 2}{2^{b}}, \ldots, \frac{2 \pi *\left(2^{b}-1\right)}{2^{b}}\right\} .
\end{aligned}
$$

To optimize the reflection coefficient matrices $\left\{\boldsymbol{\Phi}_{l}\right\}_{l=1}^{L}$, we first attempt to transform (13) to an explicit expression of SINR about $\left\{\boldsymbol{\Phi}_{l}\right\}_{l=1}^{L}$. By utilizing the sets (15) defined above, $\left|\Theta^{H}[i] \boldsymbol{p}\right|^{2}$ can be equivalently expressed as

$$
\begin{aligned}
\left|\boldsymbol{\Theta}^{H}[i] \boldsymbol{p}\right|^{2} & =\left|\sum_{m \in \mathcal{M}} \theta_{m}[i] p_{m}\right|^{2} \\
& =\left|\sum_{l=1}^{L} \sum_{m \in \mathcal{M}_{l}[i]} \boldsymbol{g}_{l}^{H} \boldsymbol{\Phi}_{l} \boldsymbol{h}_{m, l} p_{m}\right|^{2} .
\end{aligned}
$$

Let $\boldsymbol{g}_{l}^{H} \boldsymbol{\Phi}_{l} \boldsymbol{h}_{m, l} p_{m}=\boldsymbol{\varphi}_{l}^{H} \operatorname{diag}\left\{\boldsymbol{g}_{l}^{H}\right\} \boldsymbol{h}_{m, l} p_{m}, \quad \varphi_{l}^{H}=$ $\left[e^{j \varphi_{l, 1}}, e^{j \varphi_{l, 2}}, \cdots, e^{j \varphi_{l, N}}\right]=\operatorname{diag}\left\{\boldsymbol{\Phi}_{l}\right\}$ is an $1 \times N$ vector, and $\boldsymbol{v}_{m, l}=\operatorname{diag}\left\{\boldsymbol{g}_{l}^{H}\right\} \boldsymbol{h}_{m, l} p_{m}$. The equation (17) can be further expressed as

$$
\begin{aligned}
\left|\boldsymbol{\Theta}^{H}[i] \boldsymbol{p}\right|^{2} & =\left|\sum_{l=1}^{L} \sum_{m \in \mathcal{M}_{l}[i]} \boldsymbol{\varphi}_{l}^{H} \boldsymbol{v}_{m, l}\right|^{2} \\
& =\left|\boldsymbol{u}^{H} \sum_{l=1}^{L} \sum_{m \in \mathcal{M}_{l}[i]} \mathbf{E}_{l} \boldsymbol{v}_{m, l}\right|^{2},
\end{aligned}
$$

where $\boldsymbol{u}^{H}=\left[\boldsymbol{\varphi}_{1}^{H}, \boldsymbol{\varphi}_{2}^{H}, \cdots, \varphi_{L}^{H}\right]$ is an $1 \times N L$ vector which represents the configuration of all the IRSs. Define the matrix $\mathbf{E}_{l} \in \mathbb{C}^{N L \times N}$ as

$$
\mathbf{E}_{l}^{H}=\left[\mathbf{0}_{N}, \cdots, \mathbf{0}_{N}, \mathbf{I}_{N}, \mathbf{0}_{N}, \cdots, \mathbf{0}_{N}\right],
$$

where the $l$-th $N \times N$ square matrix is identity matrix and the other $L-1$ square matrices are zero matrices. Therefore, we can obtain the relationship that $\varphi_{l}^{H}=\boldsymbol{u}^{H} \mathbf{E}_{l}$. Then we substitute (18) and (19) into (16), the objective function SINR can be rewritten as

$$
\operatorname{SINR}=\frac{\left|\boldsymbol{u}^{H} \boldsymbol{V}_{0}\right|^{2}}{\sum_{i=-v, i \neq 0}^{v}\left|\boldsymbol{u}^{H} \boldsymbol{V}_{i}\right|^{2}+\sigma^{2}},
$$

where $\boldsymbol{V}_{i}=\sum_{l=1}^{L} \sum_{m \in \mathcal{M}_{l}[i]} \mathbf{E}_{l} \boldsymbol{v}_{m, l}$

Problem (16) is a multiple-ratio fractional programming problem, which can be translated to the following problem by utilizing the quadratic transform method proposed in [34]

$$
\begin{gathered}
\max _{\mathbf{u}, y} f_{1}(\boldsymbol{u}, y) \\
\text { s.t. } \varphi_{l, n} \in \mathcal{F},
\end{gathered}
$$

where the new objective function is

$f_{1}(\boldsymbol{u}, y)=2 \operatorname{Re}\left\{y^{*} \boldsymbol{u}^{H} \boldsymbol{V}_{0}\right\}-|y|^{2}\left(\sum_{i=-v, i \neq 0}^{v}\left|\boldsymbol{u}^{H} \boldsymbol{V}_{i}\right|^{2}+\sigma^{2}\right)$,

and $y$ refers to the auxiliary variable.

Based on the aforementioned analysis, solving problem (16) over $\varphi_{l, n}$ is equivalent to solving problem (21) over $\boldsymbol{u}$ and $y$. Problem (21) is a biconvex optimization problem. Generally, through alternatively updating $\boldsymbol{u}$ and $y$ by fixing one of them 
and solving the corresponding convex optimization problem can find a good solution. Optimal $y$ can be obtained with given $\boldsymbol{u}$, by setting $\partial f_{1}(\boldsymbol{u}, y) / \partial y=0$

$$
y^{o p t}=\frac{\boldsymbol{u}^{H} \boldsymbol{V}_{0}}{\sum_{i=-v, i \neq 0}^{v}\left|\boldsymbol{u}^{H} \boldsymbol{V}_{i}\right|^{2}+\sigma^{2}} .
$$

Then we consider to obtain the optimal $\boldsymbol{u}$ with given $y^{o p t}$. With the relationship that $\left|\boldsymbol{u}^{H} \boldsymbol{V}_{i}\right|^{2}=\boldsymbol{u}^{H} \boldsymbol{V}_{i} \boldsymbol{V}_{i}^{H} \boldsymbol{u}$, we can transform (21) as

$$
\begin{array}{ll}
\max _{\boldsymbol{u}} & f_{2}(\boldsymbol{u})=-\boldsymbol{u}^{H} \boldsymbol{A} \boldsymbol{u}+2 \operatorname{Re}\left\{\boldsymbol{u}^{H} \boldsymbol{b}\right\}+c, \\
\text { s.t. } & \varphi_{l, n} \in \mathcal{F} .
\end{array}
$$

where $\boldsymbol{A}=|y|^{2} \sum_{i=-v, i \neq 0}^{v} \boldsymbol{V}_{i} \boldsymbol{V}_{i}^{H}$ is an $N L \times N L$ Hermitian matrix, i.e., $\boldsymbol{A}_{q, r}=\boldsymbol{A}_{q, r}^{*}$. Moreover, $\boldsymbol{b}=y^{*} \boldsymbol{V}_{0}$ is an $N L \times 1$ vector and $c=-\sigma^{2}|y|^{2}$. Let

$$
\begin{aligned}
& \boldsymbol{A}_{q, r}=a_{q, r} \\
& \boldsymbol{u} \triangleq\left[u_{1}, \cdots, u_{n}, \cdots, u_{N L}\right]^{T} \\
& =\left[e^{-j \varphi_{1,1}}, \cdots, e^{-j \varphi_{1, N}}, \cdots, e^{-j \varphi_{L, 1}}, \cdots, e^{-j \varphi_{L, N}}\right]^{T}, \\
& \boldsymbol{b} \triangleq\left[b_{1}, \cdots, b_{n}, \cdots, b_{N L}\right]^{T} .
\end{aligned}
$$

Problem (24) can be solved by semidefinite program (SDP) method, which first relaxes the discrete IRS phase levels to continuous levels and then selects optimal results from the available phase set by nearest point projection [20]. This SDP method can not obtain a closed-form solution and is computationally expensive [28], since sufficiently large number of randomizations need to be performed to guarantee the performance [21]. In the following, we solve this problem by serially optimizing the element of $\boldsymbol{u}$.

Lemma 2: Problem (24) can be optimized by iteratively obtaining the optimal $n$-th element of $\boldsymbol{u}$ serially.

Proof: According to [20], by iteratively optimizing one element of $\boldsymbol{u}$ while keeping the others fixed, the objective value of problem (24) is non-decreasing.

Considering the $n$-th element of $\boldsymbol{u}$, we can translate problem (24) to a function of $u_{n}$

$$
\begin{array}{cc}
\max _{u_{n}} & f_{3}\left(u_{n}, y\right), \\
\text { s.t. } & \angle u_{n}^{*} \in \mathcal{F},
\end{array}
$$

where the function $f_{3}\left(u_{n}, y\right)$ is presented as

$$
\begin{aligned}
f_{3}\left(u_{n}, y\right) & =-a_{n, n}+2 \operatorname{Re}\left\{u_{n}^{*}\left(b_{n}-\sum_{r=1, r \neq n}^{N L} a_{n, r} u_{r}\right)\right\} \\
& +g\left(u_{1}, \ldots, u_{n-1}, u_{n+1}, \ldots, u_{N L}\right) .
\end{aligned}
$$

Let $d_{n}=b_{n}-\sum_{r=1, r \neq n}^{N L} a_{n, r} u_{r}=\alpha e^{j \beta}$, and $\alpha=\left|d_{n}\right|$, $\beta=\arctan \left(\frac{\operatorname{Im}\left\{d_{n}\right\}}{\operatorname{Re}\left\{d_{n}\right\}}\right), \varphi_{n}=\arctan \left(\frac{\operatorname{Im}\left\{u_{n}^{*}\right\}}{\operatorname{Re}\left\{u_{n}^{*}\right\}}\right)$, that is $u_{n}=$

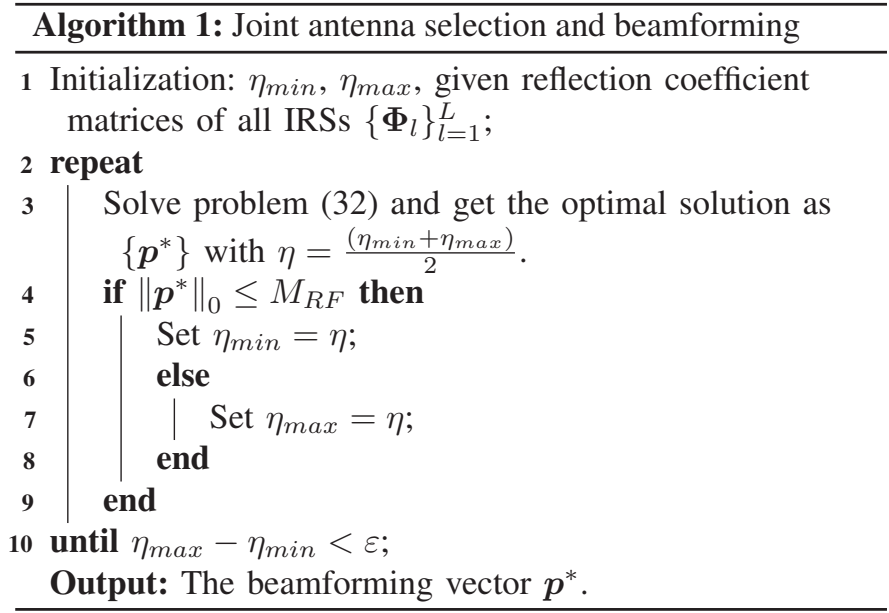

$e^{-j \varphi_{n}}$. Then problem (26) can be equivalently translated to

$$
\begin{array}{cl}
\max _{u_{n}} & f_{4}\left(u_{n}, y\right) \\
\text { s.t. } & \varphi_{n} \in \mathcal{F},
\end{array}
$$

where $f_{4}\left(u_{n}, y\right)=2 \operatorname{Re}\left\{e^{j \varphi_{n}} \alpha e^{j \beta}\right\}=2 \alpha \cos \left(\beta+\varphi_{n}\right)$. Therefore, we can obtain the optimal solution of $\varphi_{n}$ as

$$
\varphi_{n}^{\text {opt }}=\arg \min _{\varphi_{n} \in \mathcal{F}}\left|\beta+\varphi_{n}\right| .
$$

The complexity of solving (28) is the same as calculating the values of (25), which is obviously $\mathcal{O}(L N)$. This is also the complexity for obtaining one optimal phase element of the whole $L N$ elements. As a result, the complexity of IRS reflection coefficient matrices optimization is $\mathcal{O}\left(L^{2} N^{2}\right)$, which is much lower than the SDP method of $\mathcal{O}\left(L^{6} N^{6}\right)$.

\section{BS beamforming vector optimization}

With given IRS reflection coefficient matrices $\left\{\boldsymbol{\Phi}_{l}\right\}_{l=1}^{L}$, problem (14) can be translated to the following beamforming optimization problem

$$
\begin{array}{ll}
\max _{\boldsymbol{p}} \frac{\left|\boldsymbol{\Theta}^{H}[0] \boldsymbol{p}\right|^{2}}{\sum_{i=-v, i \neq 0}^{v}\left|\boldsymbol{\Theta}^{H}[i] \boldsymbol{p}\right|^{2}+\sigma^{2}} \\
\text { s.t. } \quad\|\boldsymbol{p}\|^{2} \leq P_{s}, \\
\quad\|\boldsymbol{p}\|_{0} \leq M_{R F} .
\end{array}
$$

Now we present the method of joint antenna selection and beamforming with limited RF chains constraint. By introducing a slack variable $\eta$, problem (30) can be equivalently translated as

$$
\begin{array}{ll}
\max _{\boldsymbol{p}, \eta} & \eta \\
\text { s.t. } & \frac{\left|\boldsymbol{\Theta}^{H}[0] \boldsymbol{p}\right|^{2}}{\sum_{i=-v, i \neq 0}^{v}\left|\Theta^{H}[i] \boldsymbol{p}\right|^{2}+\sigma^{2}} \geq \eta \\
& \|\boldsymbol{p}\|^{2} \leq P_{s}, \\
& \|\boldsymbol{p}\|_{0} \leq M_{R F} .
\end{array}
$$


Problem (31) is non-convex because of the non-convex constraint (31d). To solve problem (31), we first give the following Lemma 3.

Lemma 3: There exists an optimal solution $\boldsymbol{p}^{*}$, which satisfies $\Theta^{H}[0] \boldsymbol{p}^{*}=\left|\Theta^{H}[0] \boldsymbol{p}^{*}\right|$.

Proof: Assume $\boldsymbol{p}^{\text {opt }}$ is the optimal solution of (31). If the phase angle of $\Theta^{H}[0] \boldsymbol{p}^{o p t}$ is $\omega$, then we can obtain another optimal solution $\boldsymbol{p}^{*}=e^{-j \omega} \boldsymbol{p}^{\text {opt }}$. Hence $\left|\boldsymbol{\Theta}^{H}[0] \boldsymbol{p}^{\text {opt }}\right|^{2}=$ $\left|\Theta^{H}[0] p^{*}\right|^{2}$. Meanwhile, we compensate the phase angle $\omega$ by multiplying $e^{-j \omega}$ which leads to the above lemma that $\boldsymbol{\Theta}^{H}[0] \boldsymbol{p}^{*}=\left|\boldsymbol{\Theta}^{H}[0] \boldsymbol{p}^{*}\right|=\operatorname{Re}\left\{\boldsymbol{\Theta}^{H}[0] \boldsymbol{p}^{*}\right\}$

According to [16], we can first translate (31) to a feasible problem. For a given SINR target $\eta$, if the problem is feasible, then the optimal value of (31) is no smaller than the given $\eta$. Then we can utilize bisection searching [36] to increase the value of $\eta$. By solving a series of the feasibility problems over given $\eta$, the optimal solution of problem (31) can be obtained.

The feasibility problem can be equivalently tranformed to a $l_{0}$ norm minimization problem. Since the non-convex $l_{0}$ norm is difficult to be solved, we leverage the $l_{1}$ norm minimization as a convex approximation for the $l_{0}$ norm minimization. Given value $\eta$, the $l_{1}$ minimization problem can be expressed as

$$
\begin{aligned}
& \min _{\boldsymbol{p}}\|\boldsymbol{p}\|_{1} \\
& \text { s.t. }\left\|\begin{array}{c}
\boldsymbol{\Theta}^{H}[-v] \boldsymbol{p} \\
\boldsymbol{\Theta}^{H}[-v+1] \boldsymbol{p} \\
\vdots \\
\boldsymbol{\Theta}^{H}[-1] \boldsymbol{p} \\
\boldsymbol{\Theta}^{H}[1] \boldsymbol{p} \\
\vdots \\
\boldsymbol{\Theta}^{H}[v] \boldsymbol{p} \\
\sigma \\
\|\boldsymbol{p}\|^{2} \leq P_{s} .
\end{array}\right\| \leq \frac{1}{\eta} \operatorname{Re}\left(\Theta^{H}[0] \boldsymbol{p}\right)
\end{aligned}
$$

It can be seen that problem (32) is convex, which can be conveniently solved by utilizing $\ell_{1}$-norm optimization packages [37]. The detailed joint antenna selection and beamforming algorithm is presented in Algorithm 1.

Meanwhile, the two-step optimization algorithm is presented in Algorithm 2, and we give its convergence analysis as follows.

Theorem 1: For Algorithm 2, if the reflecting coefficients satisfy the following condition under the $t$-th iteration, then Algorithm 2 converges

$$
f_{2}\left(\boldsymbol{u}^{(t)}\right) \geq f_{2}\left(\boldsymbol{u}^{(t-1)}\right) .
$$

Proof: After each iteration, the BS beamforming vectors $\boldsymbol{p}^{(t)}$ will inevitably increase the SINR of the user. Therefore, if the condition (33) is satisfied, the objective function is a monotonically nondecreasing problem, which means Algorithm 2 is guaranteed to converge.

Since the optimal solution is obtained in (28), under each iteration we can obtain the optimal $\varphi_{n}^{o p t}$ by fixing other reflecting coefficients. Therefore, for $t$-th iteration in Algorithm

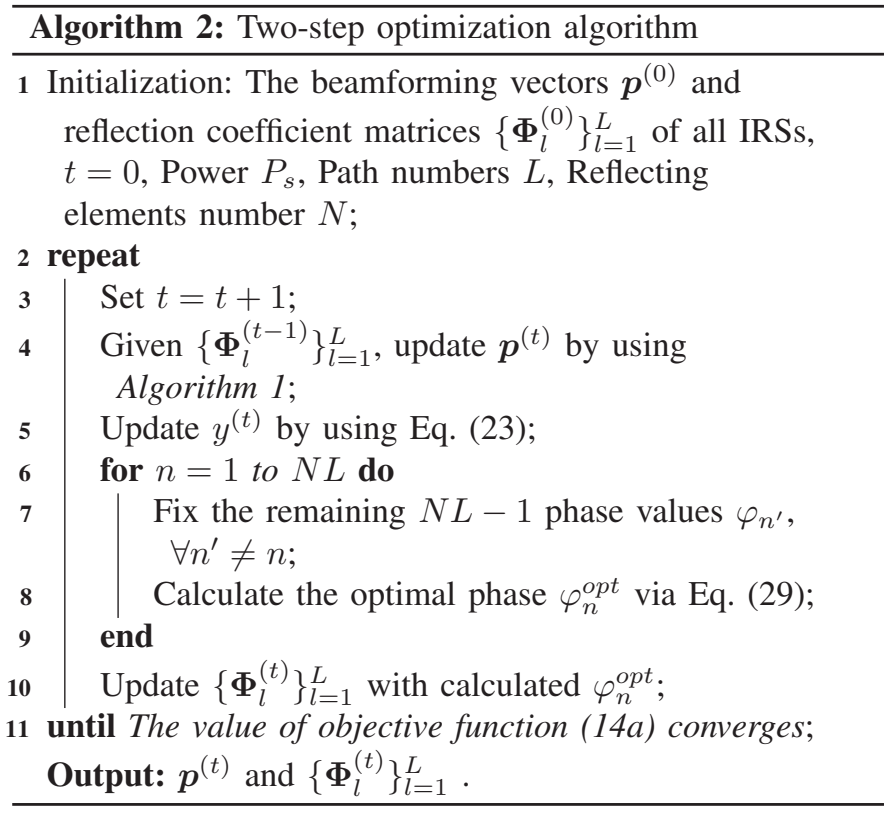

2, the updated $\boldsymbol{u}^{(t)}$ with given $\boldsymbol{u}^{(t-1)}$ will always satisfy the constraint (33) in Theorem 1. In this case, Algorithm 2 is guaranteed to converge.

Remark 3: Our proposed algorithm can be extended to the system model with the existence of direct channel links between the BS and the user, or other reflection links without involving any IRS.

Proof: See in Appendix A.

\section{Performance Evaluation}

\section{A. Simulation Scenario}

Extensive simulations are conducted to evaluate our proposed two-step algorithm. We assume the multi-IRS assisted wideband mmWave lens system operates at $28 \mathrm{GHz}$ with bandwidth $B=500 \mathrm{MHz}$. The LoS path between the BS and the user is severely blocked by obstacles, and we assume the BS-IRS mmWave channels are generated by (2). The IRS-user mmWave channels are obtained according to the geometric channel model [3]. Meanwhile, the complexvalued path gain is generated by $\beta_{l} \sim \mathcal{C N}\left(0,10^{-0.1 P L}\right)$ where $P L=\alpha+10 \beta \log _{10}(d)+\xi, \xi \sim \mathcal{N}\left(0, \sigma^{2}\right)$, and the values of these parameters follow from [39]. Furthermore, the path delays are uniformly distributed in $\left[0, T_{m}\right]$, where $T_{m}=100 \mathrm{~ns}$ represents the maximum path delay. As a reult, we have $B T_{m} \gg 1$ and the system is wideband in general. Unless otherwise stated, the simulation parameters are set as the default values given in Table I.

To show the robustness (i.e., the superior performance under various scenarios) of our proposed algorithm, we investigate the performance comparision in terms of diffirent metrics. Meanwhile, our proposed two-step optimization algorithm is compared with the following benchmark schemes:

- Scheme 1 (Fully Digital + MMSE): We consider the special case that the BS has full RF chains [14], [16], i.e., $M_{R F}=M$. The IRS reflection coefficient matrices optimization is the same as our proposed algorithm and 
TABLE I

Default Values

\begin{tabular}{lc}
\hline \hline Notation & Description \\
\hline Number of antenna elements at BS $(M)$ & 141 \\
Number of RF chains at BS $\left(M_{R F}\right)$ & 20 \\
Noise power $\left(\sigma^{2}\right)$ & $-85 \mathrm{dbm}$ \\
Transmit power constraint at BS $\left(P_{s}\right)$ & $30 \mathrm{dbm}$ \\
Number of reflect elements in each IRS $(N)$ & 50 \\
Distance between BS and user & $50 \mathrm{~m}$ \\
Phase resolution $(b)$ & 1 \\
Number of IRSs or main paths $(L)$ & 4 \\
IRSs location & $(10,-15),(15,12),(20,-7),(25,5)$ \\
BS location & $(0,0)$ \\
User location & $(50,0)$ \\
\hline \hline
\end{tabular}

the BS beamforming vector is optimized by MMSE beamforming [16].

- Scheme 2 (Distributed + MRT): The IRS reflection coefficient matrices are optimized by the distributed algorithm proposed by [21]. According to the Power Based Antenna Selection in [16], we first optimize the BS beamforming vector by the maximum-ratio transmission (MRT) beamforming [21] with $M_{R F}=M$. Then we select those $M_{R F}$ antennas with the most significant transmit power and obtain the beamforming vector by MRT beamforming.

- Scheme 3 (Distributed + MMSE): The same as Scheme 2 while MMSE beamforming is utilized for BS beamforming.

- Scheme 4 (Random + MMSE): The IRS reflection coefficient matrices are not optimized, in which the phase elements are randomly chosen from set $\mathcal{F}$ [20]. Then the BS beamforming vector is obtained by the Power Based Antenna Selection and MMSE beamforming.

- Scheme 5 (Without IRS + MRT): We generate the BSuser channel with only NLoS components taken into consideration. Then we adopt the Power Based Antenna Selection and MRT beamforming for the BS beamforming vector [28].

\section{B. Convergence Performance}

The convergence performance of the proposed algorithm is presented in Fig. 2, under different settings of phase resolutions and reflect elements for each IRS. It can be seen that, all these three curves converge to the stable solution after no more than 10 iterations. With increasing phase resolution $b$ and reflect elements number $N$, the convergence speed becomes slightly slower and converge to higher SINR. Nevertheless, our proposed algorithm can converge at an acceptable rate.

\section{Impact of IRS Parameters}

To evaluate the performance of our proposed algorithm under various IRS parameters, we evaluate the SINR versus the number of reflect elements $N$ as shown in Fig. 3.

In Fig. 3, the four curves with IRS assisted ascend as the reflect elements number $N$ increases. This is because more signals can be reflected and more effective IRS reflection coefficient matrices can be achieved when there are more

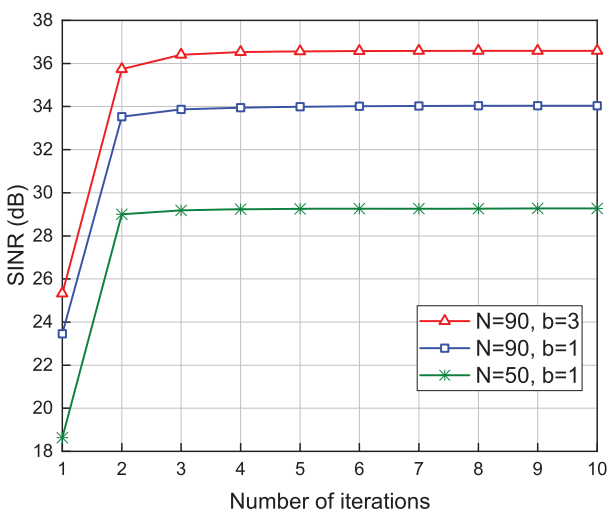

Fig. 2. Convergence performance of the proposed algorithm with different number of phase resolutions and reflect elements.

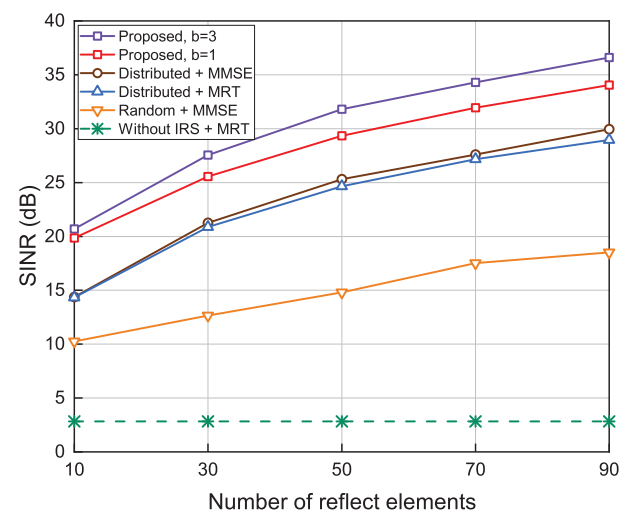

Fig. 3. SINR versus the number of reflect elements.

reflect elements in each IRS. With higher phase resolution, our proposed algorithm can achieve better performance. The reason is that with more discrete phase levels, the IRSs can steer the incident signals with finer direction and thus can achieve more performance gains. Due to the joint optimization of IRS reflection coefficient matrices and BS beamforming vector, the proposed algorithm outperforms benchmark schemes in Fig. 3. Specifically, $5 \mathrm{~dB}$ SINR gain can be achieved compared with existing algorithm in [21].

\section{Impact of BS Parameters}

To characterize the advantage of multi-IRS assisted lens system and our proposed algorithm, we compare the performance of spectrum efficiency (SE) and energy efficiecy (EE) with other schemes. The SE of the system is defined as

$$
S E=\log _{2}(1+S I N R) .
$$

Furthermore, we define the EE as the ratio between SE and the total power consumption. Specifically, it can be describe as

$$
E E=\frac{S E}{\epsilon\|\boldsymbol{p}\|^{2}+M_{R F} P_{R F}+P_{c}},
$$

where $\epsilon=\xi^{-1}, \xi$ is the power amplifier efficiency. The power consumed by each RF chain is denoted by $P_{R F}$ and 


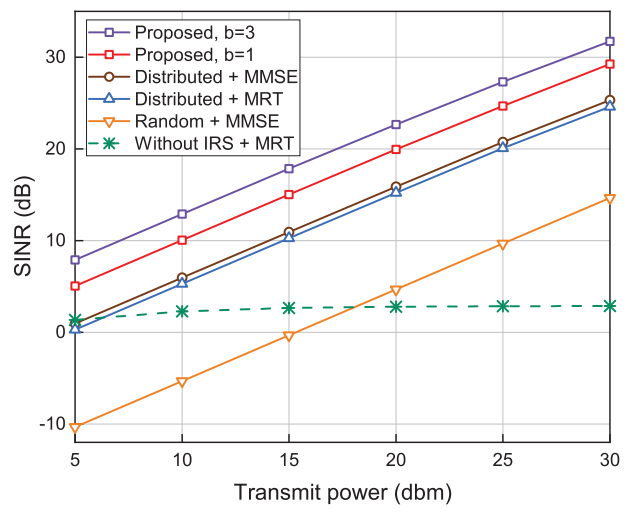

Fig. 4. SINR versus the transmit power.

$P_{c}$ represents the sum of power dissipated by all IRSs and the total circuits power consumption. We adopt the typical values $\epsilon=1.2, P_{R F}=300 \mathrm{~mW}$ and $P_{c}=300 \mathrm{~mW}$ [14]. To show the superiority of our proposed IRS and lens combined system and our proposed algorithm, we calculate the EE results of all the schemes. In addition, the EE optimization in IRS-assisted mmWave lens system can be solved by the quadratic transformation and the sequential convex approximation method [31].

In Fig. 4, we plot the SINR versus the transmit power $P_{s}$. All these curves ascend with $P_{s}$ increasing while our proposed algorithm achieves significant performance gains compared with benchmark schemes. This can be explained as follows. The MRT beamforming and the distributed algorithm [21] aim to maximize the received signal power at the user, which inevitably increases the ISI at the user. Although the MMSE beamforming takes the noise power into account, this scheme is still insufficient to achieve satisfying SINR performance since the antenna selection method and the distributed algorithm are less effective than our proposed algorithm. In addition, the random scheme [20] has no contribution to the SINR which causes the worst performance in low transmit power region.

Fig. 5(a) shows the SE versus the number of RF chains at BS. With the number of RF chains increasing, the performance gap between the fully digital scheme and our proposed algorithm becomes smaller. This is because the fully digital can be considered as a special case, i.e., $M_{R F}=M$. When the number of RF chains $M_{R F}=22$, our proposed algorithm reaches $98 \% \mathrm{SE}$ of the fully digital scheme without incurring huge energy consumption as shown in Fig. 5(b), since the BS beamforming vector with limited RF chains is efficiently optimized by our proposed algorithm. The scheme without IRSs has no SE improvement since the MRT beamforming increases both the received signal power and the ISI with $M_{R F}$ increasing, and the signal power gain brought by the increasement of $M_{R F}$ is negligible compared with the severe signal degradation caused by the blockage of the LoS channel component. Meanwhile, our proposed algorithm achieves better SE and EE than other schemes, which validates the effectiveness of our joint optimization.

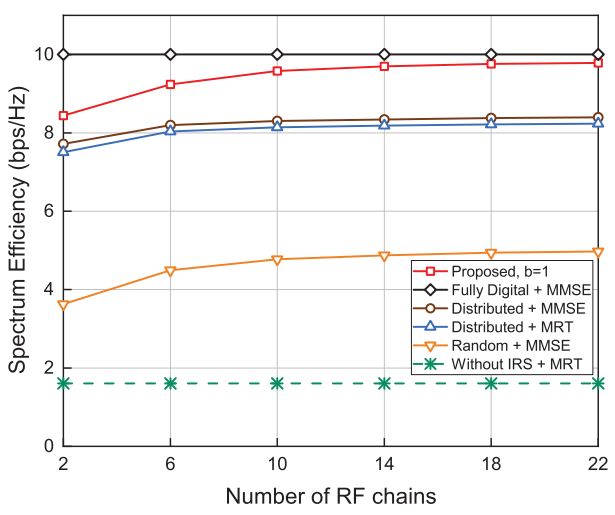

(a) Spectrum efficiency versus the number of RF chains

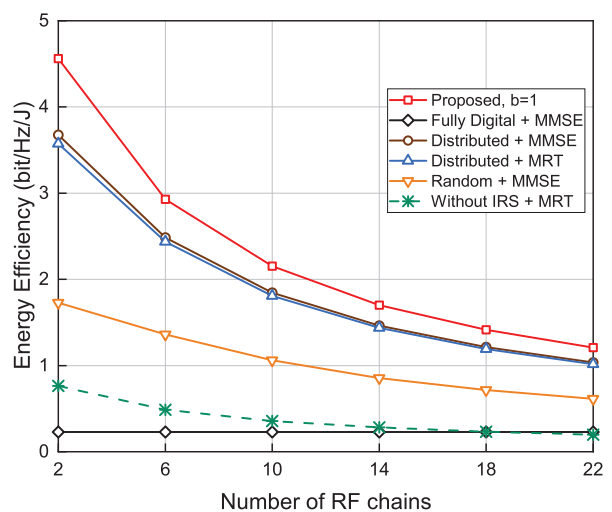

(b) Energy efficiency versus the number of RF chains

Fig. 5. The performance versus the number of RF chains.

\section{E. Impact of BS-user Distance}

The impact of the distance between the BS and the user is investigated in Fig. 6 with $L=3$ and $L=4$, respectively. For $L=3$, we remove the IRS at $(25,5)$ compared with $L=4$. As shown in Fig. 6(a), the curves with IRS increase first and begin to decrease when the BS-user distance is larger than $25 \mathrm{~m}$. For $L=3$, after removing the farthest IRS (i.e., farthest from the BS), these curves decrease from $15 \mathrm{~m}$. This difference can be explained as follow. When the user is closer to the BS compared with the IRSs at $(25,5)$ and $(20,-7)$, it is hard for these two IRS to chose appropriate phase from limited phase levels to steer the reflect signal to the user, which leads to performance degradation. The scheme without IRS suffers from severe signal degradation with only NLoS components taken into consideration, which makes the noise power comparable to the received signal power. On the other hand, the MRT beamforming improves the received signal power as well as the ISI. These factors make the curve without IRS does not decrease with distance increasing. The result verifies that our proposed algorithm achieves significant SINR gains compared with benchmark schemes under different scenarios, which further validates the robustness of our proposed system and algorithm. 


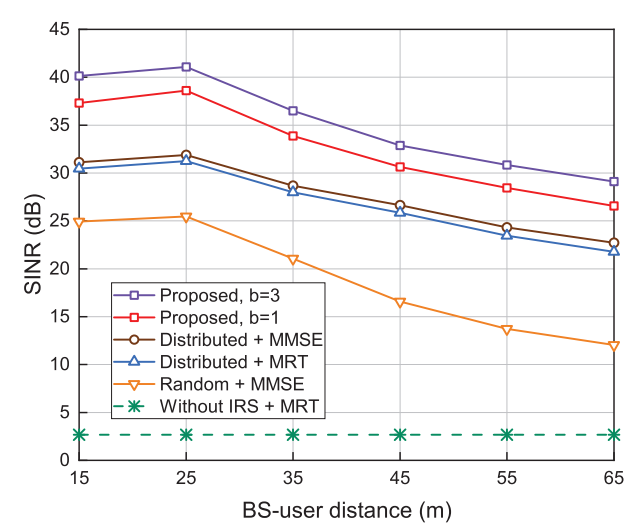

(a) SINR versus BS-user distance, $L=4$.

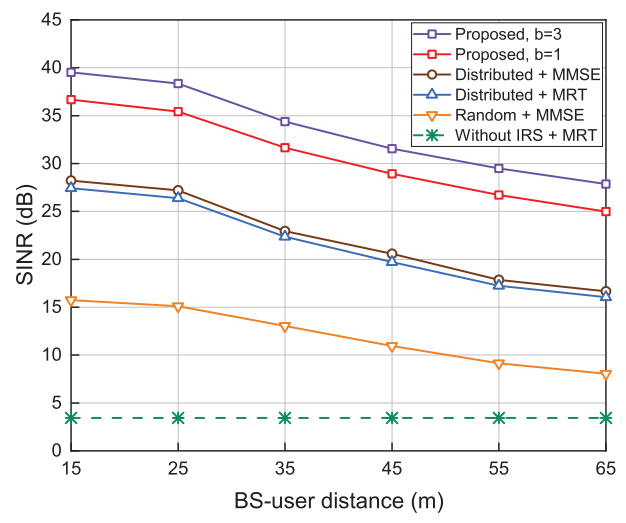

(b) SINR versus BS-user distance, $L=3$.

Fig. 6. SINR versus the distance from BS to the user.

\section{F. Outage Probability}

To further show the robustness of our proposed multi-IRS assisted lens mmWave system and our proposed algorithm, we calculate the outage probability $(\mathrm{OP})$, which is respectively defined as

$$
P_{\text {out }}\left(R_{\text {min }}\right) \triangleq \mathbb{P}\left(S E<R_{\text {min }}\right)
$$

where $R_{\min }$ denotes the required threshold. From Fig. 7, we observe that our proposed algorithm has the lowest OP compared with benchmark schemes under the same required threshold. Particularly, when $R_{\min }=7 \mathrm{bps} / \mathrm{Hz}$, the OP of our proposed algorithm reduces to zero while that of benchmarks are still larger than 0.1 .

\section{G. Complexity Discussion}

The complexity of our proposed IRS reflection coefficient matrices optimization is $\mathcal{O}\left(I_{1} L^{2} N^{2}\right)$ and that of SDP method is $\mathcal{O}\left(I_{1}^{\prime} L^{6} N^{6}\right)$, where $I_{1}$ and $I_{1}^{\prime}$ are the iteration numbers of alternatively optimizing $y$ and $\boldsymbol{u}$. Besides, the complexity of our proposed BS beamforming vector optimization is $\mathcal{O}\left(I_{2} M^{3}\right)$, where the complexity of solving (32) is generally about $\mathcal{O}\left(M^{3}\right)$ and $I_{2}$ is the iteration number for bisection searching.

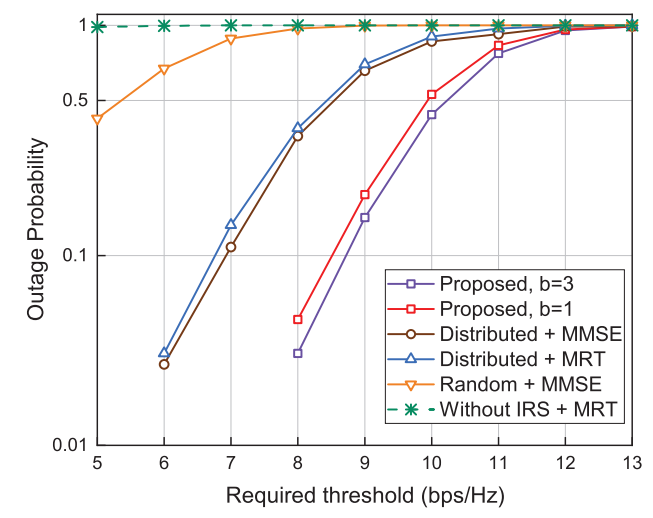

Fig. 7. Outage Probability versus required threshold.

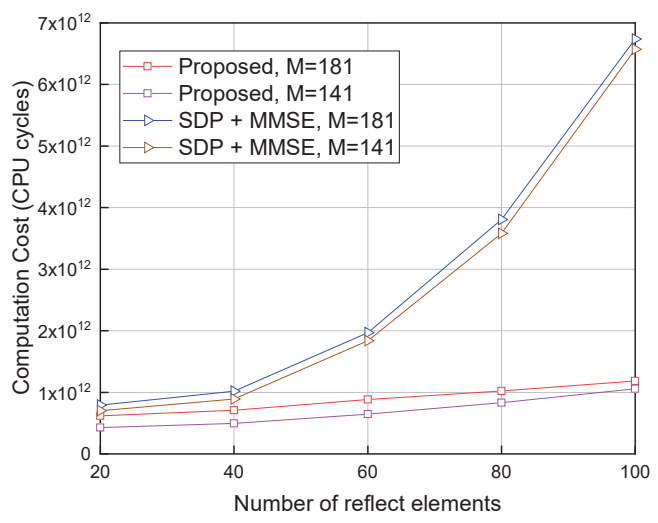

Fig. 8. Computation cost versus the number of reflect elements.

The existing method Power Based Antenna Selection in [16] requires matrix inverse operations for MMSE with complexity $\mathcal{O}\left(M^{3}+N_{R F}^{3}\right)$. Hence, the total complexity of our proposed algorithm is $\mathcal{O}\left(I_{0}\left(I_{1} L^{2} N^{2}+I_{2} M^{3}\right)\right)$ and that of SDP and MMSE combined scheme is $\mathcal{O}\left(I_{0}^{\prime}\left(I_{1}^{\prime} L^{6} N^{6}+M^{3}+N_{R F}^{3}\right)\right)$. It is hard to compare them straightforwardly in general since the complexity depends on the specific value of the parameters. If we set $N=50, M=141, N_{R F}=20, L=4, I_{0}=I_{0}^{\prime}=I_{1}=$ $I_{1}^{\prime}=I_{2}=10$, our proposed algorithm has a significantly lower complexity compared with existing methods. To further show the low-complexity of our proposed algorithm, we evaluate the CPU cycles, defined as the product of the execution time and CPU frequency. As shown in Fig. 8, with the number of reflect elements increasing, the increase of computation cost of our proposed algorithm is much smaller than that of SDP and MMSE combined scheme. Moreover, the computation cost of SDP and MMSE combined scheme becomes unaffordable when $N$ is large, while that of our proposed algorithm is still acceptable. This further proves the low-complexity and robustness of our proposed algorithm.

\section{CONClusion}

In this paper, we designed a multi-IRS assisted single-user wideband mmWave lens system to realize high robustness, low 
complexity and cost-effective mmWave communication. With the capability of steering the incident signals and reconfiguring the phase elements, IRS was utilized to overcome blockage effect, establish robust connections and enhance the performance of mmWave lens system. The SINR expression was derived by taking into account the ISI. To improve the performance of this system, a SINR maximization problem was formulated to jointly optimize beamforming vector at BS with limited RF chains and reflection coefficient matrices at IRS with discrete phase levels. To tackle it, a two-step optimization algorithm was proposed to solve the problem efficiently. Extensive simulations results demonstrated its better performance in terms of SINR and EE compared with benchmark schemes under various scenarios. Specifically, our proposed algorithm can converge at an acceptable rate and provide $5 \mathrm{~dB}$ SINR gain compared with existing algorithm.

In the future, based on the study of multi-IRS assisted SUMISO wideband lens system in this paper, it will be desirable to investigate multi-IRS assisted multi-user mmWave communication with lens antenna array, by taking the interuser interference into consideration.

\section{APPENDIX A}

\section{PROOF OF REMARK 3}

To obtain the received signal of the user with direct channel links taking into consideration, we define

$$
H_{m, l}= \begin{cases}\boldsymbol{g}_{l}^{H} \mathbf{\Phi}_{l} \boldsymbol{h}_{m, l}, & l \in\{1, \cdots, L\} \\ H_{m, 0}, & l=0 .\end{cases}
$$

where $H_{m, 0}$ represents the direct channel impulse response or other reflection links without involving any IRS between the $m$-th antenna element of the BS and the user. Then the expression of the received signal of the user can be given as

$$
\begin{aligned}
y[n] & =\sum_{m \in \mathcal{M}} \sum_{l=0}^{L} H_{m, l} x_{m}\left[n-n_{l}\right]+z[n], \\
& =\sum_{m \in \mathcal{M}} H_{m, l_{m}} p_{m} s[n] \\
& +\sum_{m \in \mathcal{M}} \sum_{l=0, l \neq l_{m}}^{L} H_{m, l} p_{m} s\left[n-\Delta_{l, l_{m}}\right]+z[n] \\
& =\boldsymbol{\Theta}^{H}[0] \boldsymbol{p} s[n]+\sum_{i=-v, i \neq 0}^{v} \boldsymbol{\Theta}^{H}[i] \boldsymbol{p} s[n-i]+z[n]
\end{aligned}
$$

where $l_{m}=\arg \max _{l=0, \ldots, L}\left\{H_{m, 0},\left(\sum_{n=1}^{N}\left|g_{l, n} h_{m, l, n}\right|\right)^{2}\right\}$ and the $m$-th element of $\boldsymbol{\Theta}^{H}[i]$ is defined as

$$
\theta_{m}[i]= \begin{cases}H_{m, l}, & \text { if } \exists l \in\{0, \cdots, L\}, \text { s.t. } n_{l}-n_{l_{m}}=i \\ 0, & \text { otherwise. }\end{cases}
$$

Then the SINR expression is the same as (13) and the BS beamforming vector optimization in Sec. III-C can be utilized. Similar to (17) and (18), $\left|\Theta^{H}[i] p\right|^{2}$ can be equivalently IEEE Transactions on Cognitive Communications and Networking expressed as

$$
\begin{aligned}
\left|\boldsymbol{\Theta}^{H}[i] \boldsymbol{p}\right|^{2} & =\left|\sum_{m \in \mathcal{M}} \theta_{m}[i] p_{m}\right|^{2} \\
& =\left|\sum_{l=0}^{L} \sum_{m \in \mathcal{M}_{l}[i]} H_{m, l} p_{m}\right|^{2} \\
& =\left|\sum_{l=1}^{L} \sum_{m \in \mathcal{M}_{l}[i]} \boldsymbol{g}_{l}^{H} \boldsymbol{\Phi}_{l} \boldsymbol{h}_{m, l} p_{m}+\sum_{m \in \mathcal{M}_{0}[i]} H_{m, 0} p_{m}\right|^{2} \\
& =\left|\boldsymbol{u}^{H} \boldsymbol{V}_{i}+\nu_{i}\right|^{2} .
\end{aligned}
$$

where $\nu_{i}=\sum_{m \in \mathcal{M}_{0}[i]} H_{m, 0} p_{m}$. With the relationship that $\left|\boldsymbol{u}^{H} \boldsymbol{V}_{i}+\nu_{i}\right|^{2}=\boldsymbol{u}^{H} \boldsymbol{V}_{i} \boldsymbol{V}_{i}^{H} \boldsymbol{u}+2 \operatorname{Re}\left\{\nu_{i}^{*} \boldsymbol{u}^{H} \boldsymbol{V}_{i}\right\}+\left|\nu_{i}\right|^{2}$, we can similarly have

$$
\begin{aligned}
& \boldsymbol{A}=|y|^{2} \sum_{i=-v, i \neq 0}^{v} \boldsymbol{V}_{i} \boldsymbol{V}_{i}^{H}, \\
& \boldsymbol{b}=y^{*} \boldsymbol{V}_{0}-|y|^{2} \sum_{i=-v, i \neq 0}^{v} \nu_{i}^{*} \boldsymbol{V}_{i}, \\
& c=-|y|^{2}\left(\sigma^{2}+\sum_{i=-v, i \neq 0}^{v}\left|\nu_{i}\right|^{2}\right)+2 \operatorname{Re}\left\{y^{*} \nu_{0}\right\} .
\end{aligned}
$$

Then the optimal solution of IRS reflection coefficient matrices can still be obtained by (29).

\section{ACKNOWLEDGMENTS}

This work was supported in part by the National Key Research and Development Program of China (2020YFA0711400) and National Science Foundation of China (61631017, 91538203).

\section{REFERENCES}

[1] J. G. Andrews, S. Buzzi, W. Choi, S. V. Hanly, A. Lozano, A. C. Soong, and J. C. Zhang, "What will 5G be?" IEEE J. Select. Areas Commun., vol. 32, no. 6, pp. 1065-1082, Apr. 2014.

[2] A. Ghosh, T. A. Thomas, M. C. Cudak, R. Ratasuk, P.Moorut, F. W. Vook, T. S. Rappaport, G. R. MacCartney, S. Sun, and S. Nie, "Millimeter-wave enhanced local area systems: a high-data-rate approach for future wireless networks," IEEE J. Select. Areas Commun., vol. 32, no. 6, pp. 1152-1163, Jun. 2014.

[3] M. R. Akdeniz et al., "Millimeter wave channel modeling and cellular capacity evaluation," IEEE J. Sel. Areas Commun., vol. 32, no. 6, pp. 1164-1179, Jun. 2014.

[4] K. Haneda, J. Jarvelainen, A. Karttunen, M. Kyro, and J. Putkonen, "A Statistical spatio-temporal radio channel model for large indoor environments at 60 and $70 \mathrm{GHz}$," IEEE Trans. Antennas Propag., vol. 63, No. 6, pp. 2694-2704, Jun, 2015.

[5] O. E. Ayach, S. Rajagopal, S. Abu-Surra, Z. Pi, and R. W. Heath, "Spatially sparse precoding in millimeter wave MIMO systems," IEEE Trans. Wireless Commun., vol. 13, no. 3, pp. 1499-1513, March. 2014.

[6] Z. Wei, L. Zhao, J. Guo, D. W. K. Ng, and J. Yuan, "Multi-Beam NOMA for Hybrid mmWave Systems," IEEE Trans. Commun., vol. 67, no. 2, pp. 1705-1719, Feb. 2019.

[7] S. Han, C. I, Z. Xu and C. Rowell, "Large-scale antenna systems with hybrid analog and digital beamforming for millimeter wave 5G," IEEE Commun. Mag., vol. 53, no. 1, pp. 186-194, Jan, 2015.

[8] Y. Zeng, R. Zhang, "Millimeter wave MIMO with lens antenna array: A new path division multiplexing paradigm," IEEE Trans. Commun., vol. 64, no. 4, pp. 1557-1571, Apr. 2016. 
[9] Y. Zeng and R. Zhang, "Cost-effective millimeter-wave communications with lens antenna array," IEEE Wireless Commun., vol. 24, no. 4, pp. 81-87, Aug. 2017.

[10] Y. Zeng, L. Yang, R. Zhang, "Multiuser millimeter wave MIMO with full-dimensional lens antenna array," IEEE Trans. Wireless Commun., vol. 17, no. 4, pp. 2800-2814, Apr. 2018.

[11] A. Sayeed and N. Behdad, "Continuous aperture phased MIMO: Basic theory and applications," in Proc. Allerton Conf. Commun., Control, Comput., pp. 1196-1203, Oct. 2010.

[12] A. M. Sayeed, "Deconstructing multiantenna fading channels," IEEE Trans. Signal Process., vol. 50, no. 10, pp. 2563-2579, Oct. 2002.

[13] J. Brady, N. Behdad, and A. M. Sayeed, "Beamspace MIMO for millimeter-wave communications: System architecture, modeling, analysis, and measurements," IEEE Trans. Antennas Propag., vol. 61, no. 7, pp. 3814-3827, Jul. 2013

[14] B. Wang, L. Dai, Z. Wang, N. Ge, S. Zhou, "Spectrum and energy efficient beamspace MIMO-NOMA for millimeter-wave communications using lens antenna array," IEEE J. Sel. Areas Commun., vol. 35, no. 10, pp. 2370-2382, Oct. 2017.

[15] R. Guo, Y. Cai, M. Zhao, Q. Shi, B. Champagne, L. Hanzo, "Joint design of beam selection and precoding matrices for mmWave MUMIMO systems relying on lens antenna arrays," IEEE J. Sel. Topics Signal Process., vol. 12, no. 2, pp. 313-325, May. 2018.

[16] W. Huang, Y. Huang, Y. Zeng, and L. Yang, "Wideband millimeter wave communication with lens antenna array: Joint beamforming and antenna selection with group sparse optimization," IEEE Trans. Wireless Commun., vol. 17, no. 10, pp. 6575-6989, Oct. 2018.

[17] J. Yang, C.-K. Wen, S. Jin, F. Gao, "Beamspace channel estimation in mmWave systems via cosparse image reconstruction technique," IEEE Trans. Commun., vol. 66, no. 10, pp. 4767-4782, Oct. 2018.

[18] M. Di Renzo, M. Debbah, D.-T. Phan-Huy, A. Zappone, M.-S. Alouini, C. Yuen, et al., "Smart radio environments empowered by reconfigurable AI meta-surfaces: an idea whose time has come," EURASIP J. Wireless Commun. Netw., vol. 2019, no. 1, p. 129, 2019.

[19] Y. Han, W. Tang, S. Jin, C.-K. Wen and X. Ma, "Large Intelligent Surface-Assisted Wireless Communication Exploiting Statistical CSI," IEEE Trans. on Veh. Technol., vol. 68, no. 8, pp. 8238-8242, Aug. 2019.

[20] H. Guo, Y. Liang, J. Chen, and E. G. Larsson, "Weighted SumRate Optimization for Intelligent Reflecting Surface Enhanced Wireless Networks," in Proc. IEEE Global Commun. Conf. (GLOBECOM) , Waikoloa, HI, USA, 2019, pp. 1-6.

[21] Q. Wu and R. Zhang, "Intelligent Reflecting Surface Enhanced Wireless Network via Joint Active and Passive Beamforming," IEEE Trans. Wireless Commun., vol. 18, no. 11, pp. 5394-5409, Aug. 2019.

[22] C. Huang, et al. "Reconfigurable intelligent surfaces for energy efficiency in wireless communication." IEEE Trans. Wireless Commun., vol. 18, no. 8, pp. 4157-4170, Aug. 2019.

[23] D. Mishra and H. Johansson, "Channel Estimation and Low-complexity Beamforming Design for Passive Intelligent Surface Assisted MISO Wireless Energy Transfer," in Proc. IEEE Int. Conf. Acoust., Speech Signal Process. (ICASSP), Brighton, U.K., May. 2019, pp. 4659-4663.

[24] C. You, B. Zheng and R. Zhang, "Channel Estimation and Passive Beamforming for Intelligent Reflecting Surface: Discrete Phase Shift and Progressive Refinement," IEEE J. Sel. Areas Commun., vol. 38, no. 11, pp. 2604-2620, Nov. 2020.

[25] B. Zheng and R. Zhang, "Intelligent Reflecting Surface-Enhanced OFDM: Channel Estimation and Reflection Optimization," IEEE Wireless Commun. Lett., vol. 9, no. 4, pp. 518-522, April. 2020.

[26] P. Wang, J. Fang, H. Duan and H. Li, "Compressed Channel Estimation for Intelligent Reflecting Surface-Assisted Millimeter Wave Systems," IEEE Signal Process. Lett., vol. 27, pp. 905-909, May. 2020.

[27] Y. Wang, H. Lu and H. Sun, "Channel Estimation in IRS-Enhanced mmWave System with Super-Resolution Network," IEEE Commun. Lett., to be published, doi: 10.1109/LCOMM.2021.3079322.

[28] P. Wang, J. Fang, X. Yuan, Z. Chen, H. Duan, H. Li, "Intelligent Reflecting Surface-Assisted Millimeter Wave Communications: Joint Active and Passive Precoding Design," IEEE Trans. Veh. Technol., vol. 69 , no. 12 , pp. $14960-14973$, Dec. 2020.

[29] J. Zuo, Y. Liu, E. Basar and O. A. Dobre, "Intelligent Reflecting Surface Enhanced Millimeter-Wave NOMA Systems," IEEE Commun. Lett., vol. 24, no. 11, pp. 2632-2636, Nov. 2020.

[30] Y. Cao, T. Lv, Z. Lin and W. Ni, "Delay-Constrained Joint Power Control, User Detection and Passive Beamforming in Intelligent Reflecting Surface-Assisted Uplink mmWave System," IEEE Trans. Cogn. Commun. Netw., Mar. 2021.

[31] Y. Wang, H. Lu, D. Zhao and H. Sun, "Energy Efficiency Optimization in IRS-Enhanced mmWave Systems with Lens Antenna Array," in Proc.
IEEE Global Commun. Conf. (GLOBECOM), Taipei, Taiwan, 2020, pp. 1-6.

[32] D. Zhao, H. Lu, Y. Wang and H. Sun, "Joint Passive Beamforming and User Association optimization for IRS-assisted mmWave Systems," in Proc. IEEE Global Commun. Conf. (GLOBECOM), Taipei, Taiwan, 2020, pp. $1-6$

[33] X. Tan, Z. Sun, D. Koutsonikolas, and J. M. Jornet, "Enabling indoor mobile millimeter-wave networks based on smart reflect-arrays," Proc. IEEE Infocom, pp. 270-278, Apr. 2018.

[34] K. Shen, W. Yu, "Fractional programming for communication systemsPart I: Power control and beamforming," IEEE Trans. on Signal Process., vol. 66, no. 10, pp. 2616-2630, 2018.

[35] L. Yang, Y. Zeng, R. Zhang, "Channel estimation for millimeter-wave MIMO communications with lens antenna arrays", IEEE Trans. Veh. Technol., vol. 67, no. 4, pp. 3239-3251, Apr. 2018.

[36] A. Wiesel, Y. C. Eldar, and S. Shamai (Shitz), "Linear precoding via conic optimization for fixed MIMO receivers," IEEE Trans. Signal Process., vol. 54, no. 1, pp. 161-176, Jan. 2006.

[37] Z. Wen and W. Yin. (2012). User Manual FPC as a MATLAB Solver for $\ell_{1}$ Regularized Least Squares Problems. [Online]. Available: https://www.caam.rice.edu/optimization/L1/FPC_AS.

[38] C. Ramirez, V. Kreinovich, M. Argaez, "Why $\ell_{1}$ is a good approximation to $\ell_{0}$ : A geometric explanation", J. Uncertain Syst., vol. 7, no. 3, pp. 203-207, 2013.

[39] M. R. Akdeniz, Y. Liu, M. K. Samimi, S. Sun, S. Rangan, T. S.Rappaport, and E. Erkip, "Millimeter wave channel modeling and cellular capacity evaluation," IEEE J. Sel. Areas Commun., vol. 32, no. 6, pp. 1164-1179, Jun. 2014.

[40] T. Yoo and A. Goldsmith, "On the optimality of multiantenna broadcast scheduling using zero-forcing beamforming," IEEE J. Sel. Areas Commun., vol. 24, no. 3, pp. 528-541, Mar. 2006.

[41] D. Tse and P. Viswanath, Fundamentals of wireless communication. Cambridge university press, 2005. 